\title{
Feeding a ROS-generator to Caenorhabditis elegans leads to increased expression of small heat shock protein HSP-16.2 and hormesis
}

\author{
Kai Hartwig · Tanja Heidler · Jan Moch • \\ Hannelore Daniel $\cdot$ Uwe Wenzel
}

Received: 16 October 2008/Accepted: 10 February 2009/Published online: 28 February 2009

(C) Springer-Verlag 2009

\begin{abstract}
Reactive oxygen species (ROS) are thought to be a driving force in the aging process. In transgenic Caenorhabditis elegans expressing green fluorescent protein (GFP) under control of the $h s p$-16.2 promoter (CL2070) 100 $\mu \mathrm{M}$ of the ROS-generator juglone induced GFP-expression. This was associated with translocation of DAF-16 to the nucleus as visualized in a transgenic strain expressing a DAF-16::GFP fusion protein (TJ356) and with increased cellular levels of reduced glutathione. RNA-interference for DAF-16 in CL2070 blocked the juglone-induced HSP-16.2 expression and the increase in glutathione levels. Higher concentrations of juglone did not further increase the adaptive responses but caused premature death, indicating hormetic adaptations unless the stressor exceeds the intrinsic protective capacity. The addition of the ROS-scavenger ascorbic acid finally blocked lifespan reductions and all of the adaptations to juglone stressing that ROS are indeed the molecular species that require protective response.
\end{abstract}

Keywords Caenorhabditis elegans - Hormesis · Reactive oxygen species - Aging - Heat shock protein . Glutathione

K. Hartwig · T. Heidler $\cdot$ H. Daniel

Department of Food and Nutrition,

Molecular Nutrition Unit,

Technical University of Munich,

Am Forum 5, 85350 Freising, Germany

J. Moch · U. Wenzel ( $\square)$

Molecular Nutrition Research,

Interdisciplinary Research Center,

Justus-Liebig-University of Giessen,

Heinrich-Buff-Ring 26-32, 35392 Giessen, Germany

e-mail: uwe.wenzel@ernaehrung.uni-giessen.de

\section{Introduction}

The exposure to high amounts of reactive oxygen species (ROS), as occurs in many tissues, e.g. by heavy physical exercise, is answered by prominent adaptive responses of the antioxidant defense system, which is of vital importance in the protection against oxidative stress [13]. Small heat-shock proteins (HSPs), a family of low-molecular weight polypeptides that are highly conserved from yeast to humans [6,34], appear of general importance for adaptations as their expression correlates usually well with the presence of various stressors [21, 31]. In the nematode Caenorhabditis elegans levels of small HSP-16.2 on the first day of adult life predicts as much as a fourfold variation in subsequent survival [29]. This correlation was highly specific for HSP-16.2 since the induction of other genes normally activated by stress did not show immediate relationship to longevity [29]. Moreover, HSP-16.2 expression was identified as a good predictor of the ability to withstand a lethal thermal stress [29] and its levels correlate well with the hormetic effects in response to heat treatment [26]. Mechanistically, HSP16 could affect lifespan by reducing oxidative stress through raising the pool of reduced glutathione (GSH) in the worm as suggested by Walker and Lithgow [35]. In a similar fashion HSP-27 is thought to mediate its effects in murine cells $[23,27]$. This protection against oxidative stress appears to be accomplished by small HSPs through a glucose-6-phosphate dehydrogenase-dependent increase in NADPH generation needed to maintain GSH in its reduced form via the GSSG-reductase and by using this redox modulator as an essential cofactor of their in vivo chaperone activity against oxidized proteins [27]. This function of HSP-16.2 in stress resistance and longevity is embedded into the insulin/IGF-1 signaling pathway that 
has been shown in many studies to be a central determinant of lifespan in C. elegans [30]. The DAF-16 transcription factor, a downstream target of the insulin/ IGF-1 receptor DAF-2 that translocates into the nucleus in the absence of insulin/IGF-1 signaling, is essential for maximal HSP-16 expression and for lifespan extension conferred by it [35]. Besides the $h s p-16.2$ gene, however, sod-3 coding for a superoxide dismutase (SOD) and $c t l-1$ and $c t l-2$ coding for catalase isoforms are further transcriptional targets of DAF-16 that contribute to the longevity phenotype of $d a f-2$ mutants, probably by preventing or repairing oxidative macromolecular damage, and knock-down of those genes by RNA-interference (RNAi) reduces the lifespan in daf-2 mutants by $10-20 \%$ $[24,25]$.

In the present study we assessed whether application of juglone, a generator of superoxide anion radicals [4], delivered via the oral route triggers the stress response in C. elegans and in how far the adaptations to juglone enable the maintenance of a normal lifespan. Nematodes were grown from the L4 stage on in an adapted liquid axenic medium to assure the proper oral uptake and transport of juglone through pumping in the worm pharynx posteriorly [2]. Induction of HSP-16.2 was estimated by the expression of green fluorescent protein (GFP) in a $h s p-16.2 p:: g f p$ transgenic strain (CL2070) and translocation of DAF-16 was visualized using a daf-16::gfp transgenic strain (TJ356). Activities of SOD, catalase and levels of reduced (GSH) and oxidized (GSSG) glutathione were determined by UV-absorbance based assays.

\section{Methods}

Nematode strains, maintenance and mass culture

Nematodes were grown on NGM agar plates seeded with E. coli strain OP50 as described [3] and were kept at $20^{\circ} \mathrm{C}$. Strains used in this study were: N2, variation bristol (wildtype); CL2070, dvIs70 Is[hsp-16.2::gfp; rol-6(su1006)]; and TJ356, zIs356 Is[daf-16::daf-16-gfp; rol-6]. To obtain synchronous populations worms were prepared as described [32]. For all experiments synchronous populations gained by bleaching were used. Only for lifespan analysis worms were picked and allowed to lay eggs to avoid stress conditions. Worms were kept at $20^{\circ} \mathrm{C}$ in CeHR medium (20; http://www.usacehr.org/cehr medium.htm) containing 30\% DYT (1.6\% casein hydrolysate, $1 \%$ bacto yeast extract, $85.5 \mathrm{mM}$ sodium chloride). OP50 and stocks were provided by the $C$. elegans Genetics Center, CGC, University of Minnesota, Minneapolis, USA.
Lifespan analysis

Synchronous populations were obtained by allowing 50 hermaphrodites to lay eggs for $3 \mathrm{~h}$. Eggs were placed on $50 \mathrm{~mm}$ NGM plates seeded with E. coli OP50 (40-50 per plate, 80-300 individuals per experiment) and grown at $20^{\circ} \mathrm{C}$ until L4 larval stage. Each population was examined for synchrony at the beginning of the reproductive period.

Lifespan analysis on agar plates with $E$. coli as the food source was carried out according to standard protocols [17]. After reaching the young adult stage worms were transferred to new NGM agar plates with growth arrested E. coli, which were treated before with $100 \mu \mathrm{g} / \mathrm{ml}$ ampicillin. Worms were monitored every day and transferred to fresh plates every third day. They were scored as dead when no response to a mechanical stimulus or no pharynx contraction was visible. Animals that crawled off the plates were replaced by a new worm from a substitution plate.

For lifespan analysis in liquid axenic medium L4 larvae were transferred into wells containing CeHR medium and were distributed into new wells after reaching young adult stage. Juglone was applied in 92\% ethanol, $8 \%$ Tween 80 (v/v) and diluted 1:100 in the final medium. Controls were always exposed to the same amount of solvent. Worms were moved to wells containing fresh medium and compounds every third day with a glass capillary and scored as dead when they did not respond to a mechanical stimulus or no more pharynx contraction was seen. Experiments were started with 70 worms per group and ten worms as a reserve for lost or bagged individuals.

\section{Stress resistance}

For measuring resistance of CL2070 to heat stress nematodes were grown in NGM medium at $20^{\circ} \mathrm{C}$. Therefore, approximately ten L1 larvae stage worms in $10 \mu \mathrm{l}$ M buffer were dispensed to each well of a 96-well flatbottomed tissue culture plate together with $40 \mu \mathrm{l}$ of resuspended OP50 bacterial culture with $\mathrm{OD}=1$. After reaching the young adult stage nematodes were incubated for $20 \mathrm{~h}$ at $20^{\circ} \mathrm{C}$ in the absence (control) or presence of either $250 \mu \mathrm{M}$ juglone or $250 \mu \mathrm{M}$ juglone plus $250 \mu \mathrm{M}$ ascorbic acid. Subsequently, worms were washed twice with M9 before single nematodes were transferred to 384well plates. Sytox Green nucleic acid stain (Invitrogen, Karlsruhe, Germany), that penetrates only in cells with compromised plasma membrane and gets fluorescent after DNA binding, was added in a final concentration of $2 \mu \mathrm{M}$. Fluorescence was measured at $535 \mathrm{~nm}$ over $24 \mathrm{~h}$ every $30 \mathrm{~min}$ after excitation at $488 \mathrm{~nm}$ using a fluorescence microtiter plate reader (Fluoroskan Ascent FL, Thermo Electron, Dreieich, Germany) that was tempered to $37^{\circ} \mathrm{C}$. 
Increases of fluorescence values twofold above the background indicated the death of the nematodes as verified by touch provocation in an independent assay.

\section{RNA-interference}

RNA-interference experiments were done as previously described using bacteria from the Ahringer deletion library [16]. Bacterial RNAi feeding strains were seeded on NGM agar plates plus $25 \mathrm{mg} / \mathrm{ml}$ carbenicillin and $1 \mathrm{mM}$ isopropyl-beta-D-thiogalactopyranoside to induce dsRNA expression at $37^{\circ} \mathrm{C}$ for $4 \mathrm{~h}$. Before usage plates were cooled down to $20^{\circ} \mathrm{C}$ and $\mathrm{L} 1$ worms were put onto plates. After reaching L4 or young adult stage worms were washed off the plates with M9 buffer and transferred into liquid medium for further experiments. Efficiency of RNAi was controlled by RT-PCR. Therefore, mRNA was extracted from 5,000 to 15,000 synchronized worms (RNAeasy; Qiagen, http://www1.qiagen.com). Unique primer pairs recognizing only cDNA derived from endogenous daf-16 mRNA were designed to avoid cross-reaction with genomic DNA and bacterially generated dsRNA and were as follows: 5'GAGACGACTACAAAGGCT3' and $5^{\prime}$ GTTCGGGGACGGAAAGA $3^{\prime}$. Relative amounts of mRNA-levels for DAF-16 versus the vector control were 0.15 .

\section{Confocal microscopy}

GFP-expression in CL2070 was analyzed at different time points as given in the figure legends and GFP-localization in TJ356 after $3 \mathrm{~h}$ of incubation in CeHR-medium containing the indicated concentrations of juglone. Thereafter, nematodes were anesthetized by the use of $5 \mathrm{mM}$ levamisole and a drop of the worm pellet was put on a glass slide pre-equipped with a thin layer of $2 \%$ agarose gel to avoid desiccation of the worms. Fluorescence was viewed under a confocal laser scanning microscope (Leica TCS SP2) with excitations at $488 \mathrm{~nm}$ and emissions at $500-530 \mathrm{~nm}$ and fluorescence intensities were determined by use of the Leica Confocal Software, version 2.5. Each experiment was repeated at least three times and ten worms per group were used in each experiment.

SOD- and catalase-activities

For activity measurements of SOD and catalase worms were incubated with or without juglone (control) in CeHRmedium for 1 day or 5 days, respectively, in the presence of $50 \mu \mathrm{M} 5$-fluorodeoxyuridine (FuDR) to prevent production of progeny. Thereafter whole worm lysates were prepared by pelleting the worms before they were resuspended in native lysis buffer and cracked open by liquid nitrogen grinding with following sonication. The protein content was measured by the BioRad protein assay (BioRad, Munich, Germany). Activities of SOD and catalase were determined by UV-absorbance measurements in a Uvicon 930 photometer (Kontron Instruments) as described [1, 22]. In brief, SOD-measurement was based on pyrogallol autoxidation at a wavelength of $420 \mathrm{~nm}$, which measures overall SOD-activity but does not differentiate between $\mathrm{Cu}-, \mathrm{Zn}-$, and $\mathrm{Mn}$-dependent isoforms. Catalase was determined by the rate of disappearance of hydrogen peroxide at $240 \mathrm{~nm}$.

\section{Glutathione-levels}

For GSH-measurements nematodes were incubated for $48 \mathrm{~h}$ in CeHR-medium with $50 \mu \mathrm{M}$ FuDR to prevent progeny. Subsequent to the incubation worms were washed several times with M9 buffer and whole worm lysates were prepared as described above. GSH and GSSG were quantified as described [28] by measuring the absorbance at $412 \mathrm{~nm}$ after reduction of 5,5'-dithio-2-nitrobenzoic acid (DTNB, Sigma, Steinheim, Germany). For the GSSG measurement glutathione has to be derivatized first by 2-vinylpyridine following the same measurement like for the total glutathione analysis. The GSH content was calculated by the difference of total glutathione and the GSSG content.

\section{Statistical analyses}

For statistical analysis Gaphpad Prism 4.01 software was used. Analysis of variance in group comparisons was performed by One-way ANOVA and significance of differences between groups were determined by a Tukey's multiple comparison test. For comparison of survival curves the Log rank test was used with $P$ values $<0.05$ being significant. Kaplan-Meier survival curves are shown in lifespan and heat stress survival figures.

\section{Results}

Although $C$. elegans appears as a perfect model to study nutrient-gene-interactions due to the ease of genetic modifications and the availability of numerous mutant strains, modifications of its nutritional environment are hard to achieve when they are grown on agar plates with $E$. coli as a food source. Dissolving compounds on agar plates and the bacterial lawn seems not to be the method of choice as the cuticula of the animals limits penetration by simple diffusion and ingestion via the bacteria seems also variable. When young adult CL2070 nematodes carrying a $h s p$ 16.2::gfp transgene were exposed to $100 \mu \mathrm{M}$ juglone either 
on agar plates with $E$. coli and the ROS-generator or in liquid axenic medium with dissolved juglone it became evident that activation of the $h s p-16.2$ driven GFP expression is much more consistent and much higher in liquid medium (Fig. 1a). The dose-response curve for the activation of the $h s p-16.2$ promoter was maximal at $4 \mathrm{~h}$ subsequent to juglone exposure (Fig. 1b) in a concentration range of $40-100 \mu \mathrm{M}$ juglone whereas at higher concentrations GFP-expression declined again (Fig. 1c). Moreover, repeated application of $100 \mu \mathrm{M}$ juglone every second day over 8 days always caused GFP-expression to a similar extent (not shown) suggesting that the decline in fluorescence observed after $4 \mathrm{~h}$ (Fig. 1b) is due to diminished ROS-levels and not to a down-regulation of the stress response during this period of life.

Previous work in C. elegans suggests that keeping animals in liquid axenic media slows development, declines fecundity, increases lifespan, decreases lipid and protein stores, and changes gene expression relative to those held on a bacterial diet. Many of these alterations mimic the consequence of dietary restriction and therefore we altered the standard liquid culture medium with respect to an optimisation of median lifespans for wildtype animals grown in liquid culture as compared to wildtype C. elegans grown on agar plates with E. coli (Fig. 2a). However,
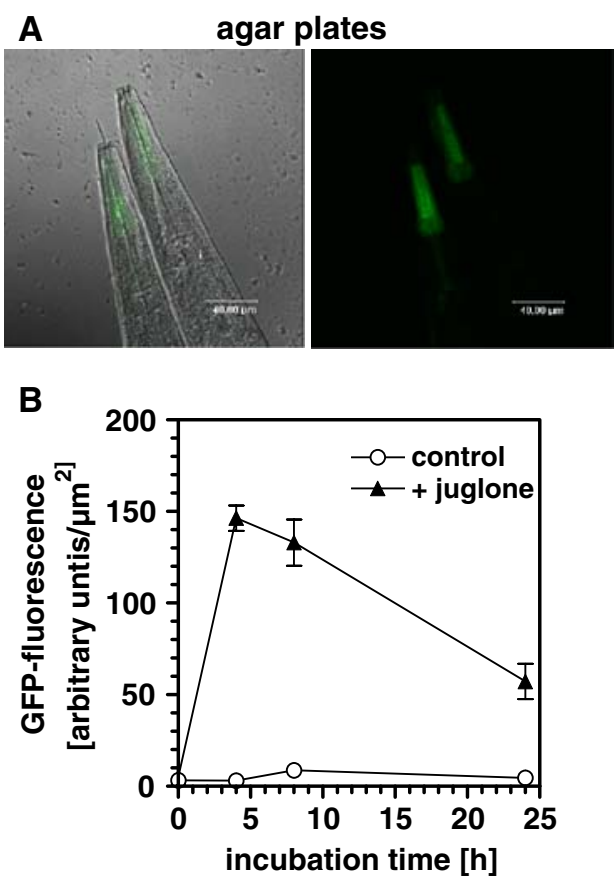

Fig. 1 HSP-16.2 expression as visualized by GFP-fluorescence in a $h s p-16.2 p::$ GFP transgenic strain (CL2070). Green fluorescence of GFP in CL2070 exposed for $4 \mathrm{~h}$ to $100 \mu \mathrm{M}$ juglone either on agar plates with E. coli as food source (left panel) or in axenic liquid culture medium (right panel) can be visualized from the fluorescence images as given in (a), left panels, and the overlays of fluorescence despite the fact that median life spans were very similar, maximum lifespan was drastically increased in axenic liquid medium (Fig. 2a). This suggests an important role for bacterial products in limiting the maximal lifespan when animals are grown on agar plates with $E$. coli.

Interestingly, CL2070 displayed a highly prolonged lifespan when compared to wildtype animals in the axenic culture medium (Fig. 2b) most likely by an integration of the transgene into lifespan-determining loci of the genome. When young adult CL2070 were exposed to $100 \mu \mathrm{M}$ juglone their lifespan curves remained unaffected (Fig. 2b), indicating that the animals were able to cope with the oxidative stress under these conditions. Concentrations of $250 \mu \mathrm{M}$ juglone, however, caused a fast decline in the numbers of animals alive (Fig. 2b).

Assuming that the increase in fluorescence in hsp$16.2 p:: g f p$ transgenic CL2070 animals in the presence of juglone (Fig. 1b, c) was dependent on DAF-16, we determined DAF-16 nuclear translocation in the transgenic strain TJ356 that expresses a DAF-16::GFP fusion protein. Both, 100 and $250 \mu \mathrm{M}$ juglone caused GFP-fluorescence to disappear from the cytosol with a concomitant accumulation of staining in the nuclei (Fig. 3a). Moreover, in the CL2070 strain, juglone-induced $h s p-16.2$ promoter driven GFP expression was completely blocked when DAF-16
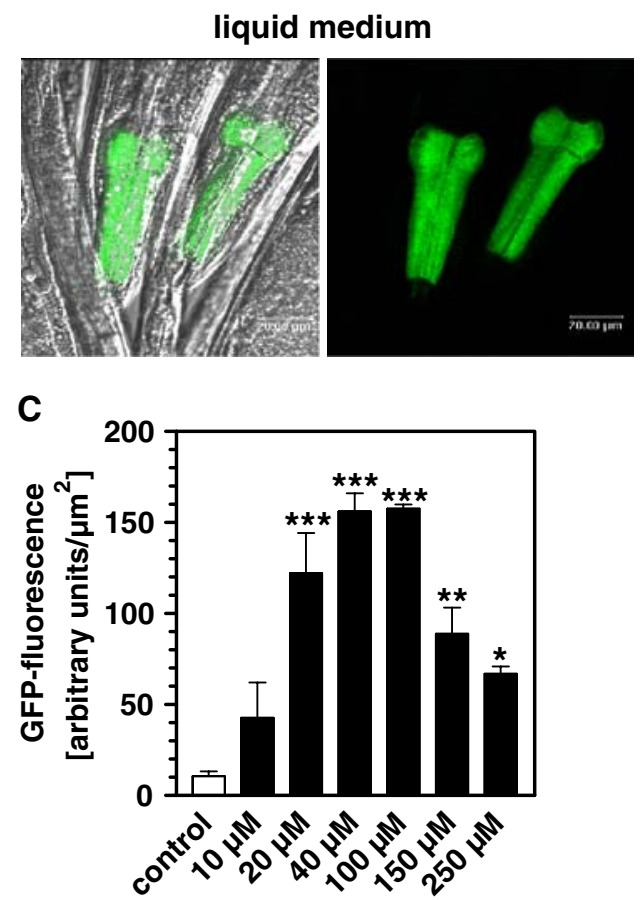

and light microscopy images (a, right panels). Time-dependent GFPfluorescence is shown in (b) when CL2070 nematodes were exposed to $100 \mu \mathrm{M}$ juglone in liquid axenic medium. Intensities of GFP expression in CL2070 cultured in liquid axenic medium for $4 \mathrm{~h}$ at various juglone concentrations is shown in (c). $* P<0.05$, $* * P<0.01, * * * P<0.001$ versus the control 

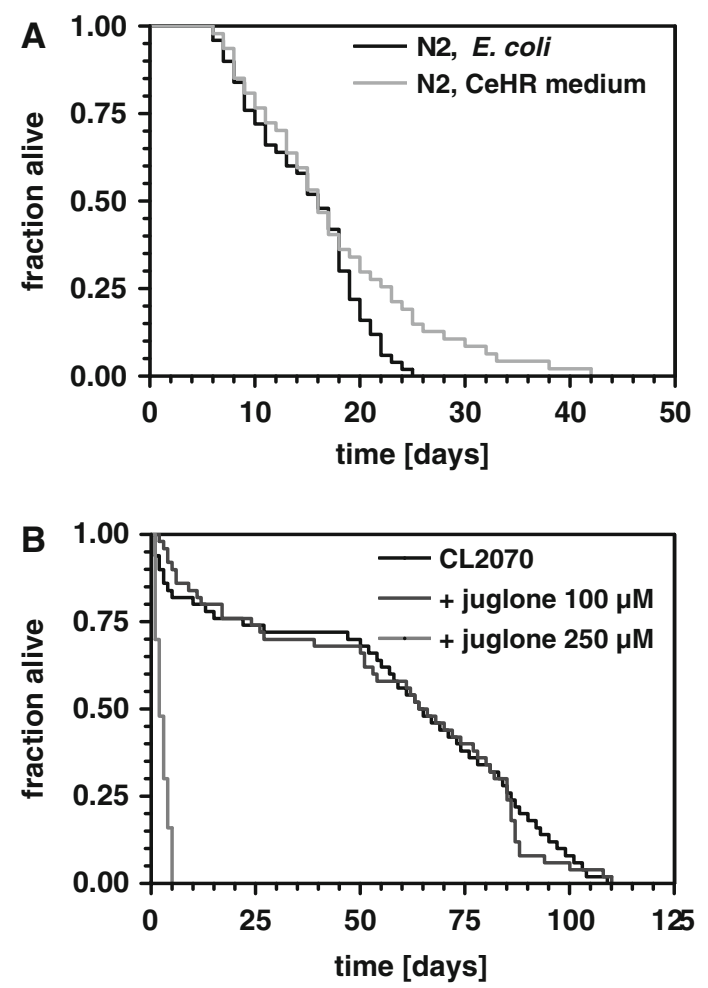

Fig. 2 Lifespan curves of N2 wildtype animals grown on agar plates with $E$. coli as a food source in comparison to those measured in liquid axenic medium is shown in (a). Lifespans of CL2070 in axenic liquid medium either in the absence (control) or presence of 100 or $250 \mu \mathrm{M}$ juglone are given in (b). $P$-values for significances of differences of survival curves were $P=0.7907$ for $100 \mu \mathrm{M}$ juglone and $P<0.0001$ for $250 \mu \mathrm{M}$ juglone versus the untreated CL2070 nematodes

was knocked-down by RNAi (Fig. 3b). The knock-down of DAF-16 blocked also the increase in GSH-levels in CL2070 upon exposure of animals to 100 or $250 \mu \mathrm{M}$ of juglone (Fig. 4a, b), whereas GSSG-levels remained unaffected by juglone (Fig. 4c). Overall, the results indicate that nuclear DAF-16 is inevitable for a proper ROS mediated stress response and that the most important downstream targets that determine resistance to ROS are the elevated GSH-levels but not the other DAF-16 targets, such as SOD or catalase that did not change in activity upon juglone treatment in CL2070 (Fig. 4c, d).

In order to demonstrate that the adaptive responses to juglone are indeed a consequence of oxidative stress ascorbic acid was applied at $250 \mu \mathrm{M}$ to scavenge ROS as caused by $250 \mu \mathrm{M}$ juglone. Ascorbic acid blocked the juglone-induced nuclear translocation of DAF-16, hsp16.2 promoter activation, and increase in GSH-levels (Fig. 5a-c). Moreover, ascorbic acid almost completely abolished the survival reducing effect of preincubation with $250 \mu \mathrm{M}$ juglone in a subsequent heat resistance assay (Fig. 5d).

\section{Discussion}

Caenorhabditis elegans has proven to be a suitable model for studying the genetic control of lifespan. Aging research in C. elegans is mainly driven by the availability of the entire genome sequence, the adaptation of RNAi technology for genome-wide loss-of-function analysis [5] and the availability of numerous mutant strains that help to define the role of distinct genes in physiological processes [8]. It seems particularly intriguing to take these concepts further and assess changes in the nutritional environment on basis of different genetic backgrounds. So far, however, studies in C. elegans have almost exclusively utilized feeding regimes in which animals are kept on agar plates with an E. coli lawn. Metabolism of supplied nutrients or nonnutritional components by the bacteria and production of mainly unknown metabolites that might contribute to the biological effects of a dietary ingredient is a concern [33]. The use of liquid axenic media on the other hand produce phenotypic changes in development, fecundity, lifespan and metabolism when compared to the apparent "gold standard" of feeding bacteria $[10,33]$. It was shown that these alterations are mainly caused by a dietary restriction and mediated by mechanisms which are distinctly different from those specified by the insulin/IGF-1 signaling pathway $[9,11]$. In the present study we report findings with an optimised liquid axenic medium that is obviously not causing a dietary restriction phenotype as judged on identical median lifespans of wildtype worms grown on either agar plates with $E$. coli or in liquid culture without bacteria. Maximum lifespan, however, was found to be prolonged in the axenic medium. It has been proposed that an increased susceptibility to bacterial infections or bacterial products in older animals when fed on bacteria may cause death [7] and the lack of these compromising conditions under axenic feeding may cause the extension of maximal life span.

We here assessed the effects of the ROS-generator juglone supplied per os on C. elegans lifespan in axenic medium. The use of CL2070 expressing GFP under control of the $h s p-16.2$ promoter [20] allowed the simultaneous visualization of stress response. CL2070 showed an unexpected threefold increase in median, average and maximum lifespan compared to N2 wildtype animals in axenic medium. CL2070 transgenic animals were generated by microinjection of a plasmid carrying the $h s p-16.2 p:: g f p$ construct along with a rol-6 morphological marker plasmid into the gonads of wildtype C. elegans [20]. Since N2 wildtype displayed identical median lifespan in our axenic medium and on agar plates and since the transgene in CL2070 is chromosomally integrated [20] our findings suggest an integration of the plasmid into gene sequences that contribute to life span regulation which in turn causes 

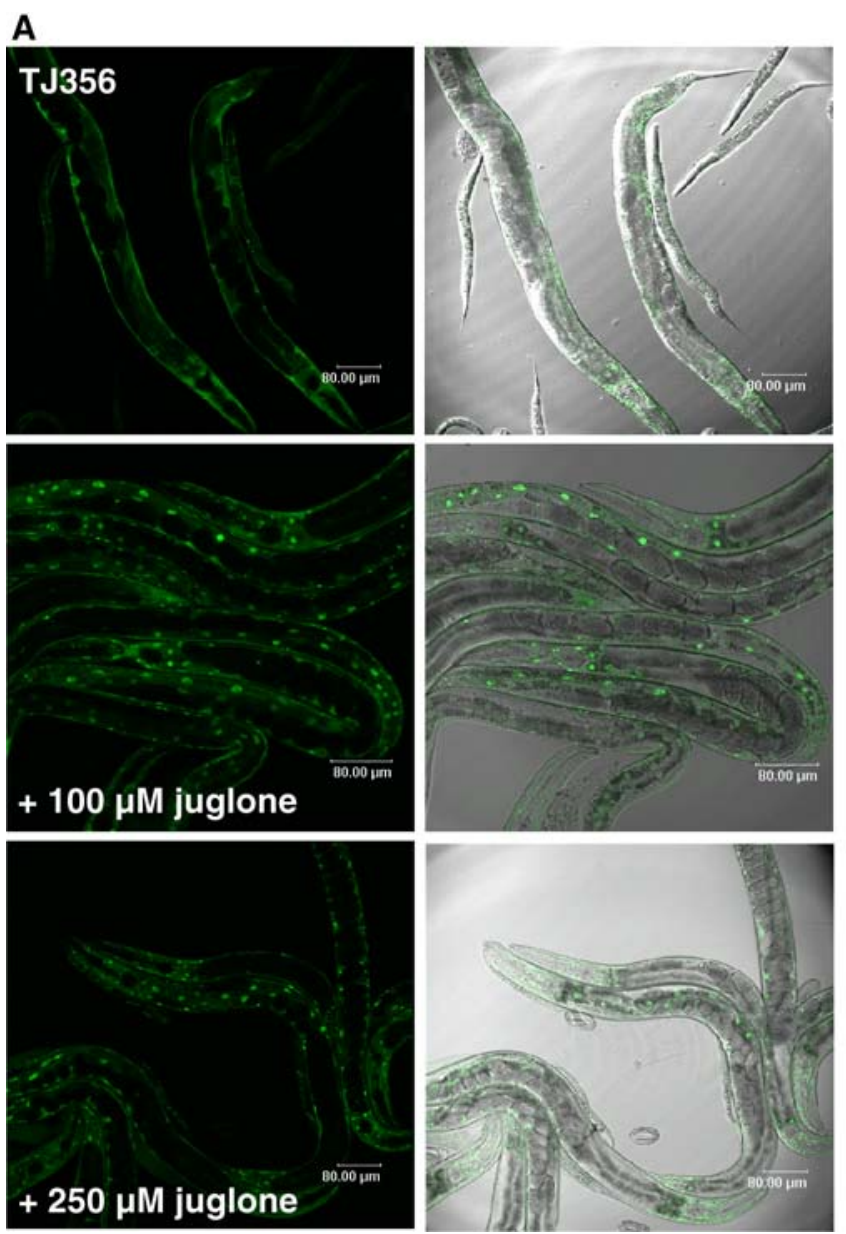

Fig. 3 HSP-16.2 expression is dependent on DAF-16. In TJ356 DAF-16 localization in the nucleus is increased by 100 and $250 \mu \mathrm{M}$ juglone (a). Knock-down of DAF-16 by RNA-interference abolished juglone-induced activation of the hsp-16-2 promoter in CL2070 as is

this longevity phenotype of CL2070. Independent on this basic longevity phenotype of CL2070, the animals show a hormetic stress response allowing the worms to cope properly with the increased ROS challenge caused by juglone concentrations of up to $100 \mu \mathrm{M}$ whereas at $250 \mu \mathrm{M}$ premature death was observed. The expression of the small heat shock protein HSP-16.2 increased in a dose dependent fashion as a function of increasing juglone concentrations with a maximal response observed at $100 \mu \mathrm{M}$ juglone. At $250 \mu \mathrm{M}$ juglone HSP-16.2 driven GFP fluorescence decreased again in parallel with premature death most likely caused by increased oxidative damage that prevents a further hormetic adaptation. HSP-16.2 levels have been shown to correlate with life expectancy in an isogenic C. elegans population [29] and our data confirm that the expression of this heat shock protein can also act as a good ROS-sensor.

The HSP-16.2 dose-dependence to juglone exposure is mimicked by HSP-16.2 adaptations to increasing
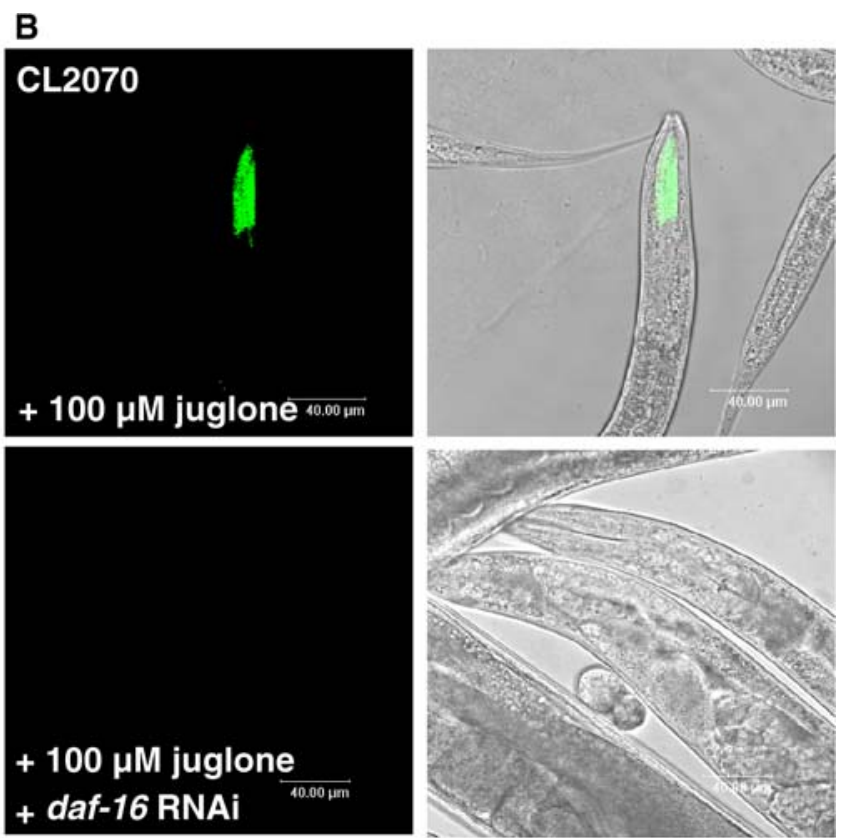

evidenced by the lack of GFP-fluorescence in (b). Left panels show the fluorescence images and right panels an overlay of fluorescence and light microscopic images

temperatures $[14,15]$. Expression of HSP-16.2 in response to the ROS-challenge is mediated by the transcription factor DAF-16 since knock-down of DAF-16 by RNAi completely abrogated GFP-fluorescence in CL2070. Moreover, juglone causes DAF-16 translocation from cytosol into the nucleus and here DAF-16 can promote transcriptional activation of HSP-16.2 expression. Our results emphasize the crucial role of DAF-16 in induction of small HSPs, which in turn can promote longevity and that this stress response pathway initiated by ROS obviously follows the same pathways as shown for heat stress [12] or hypertonic stress [18]. One of the major targets of HSP-16.2 are enzyme systems that control cellular GSHlevels. It has been shown in several mammalian cell lines that expression of different small HSPs increased the cellular contents of GSH and this response seems essential for the protective activity of these proteins against multiple stressors [23, 29]. We also observed a two-fold increase in the levels of GSH that followed the induction of HSP-16.2 

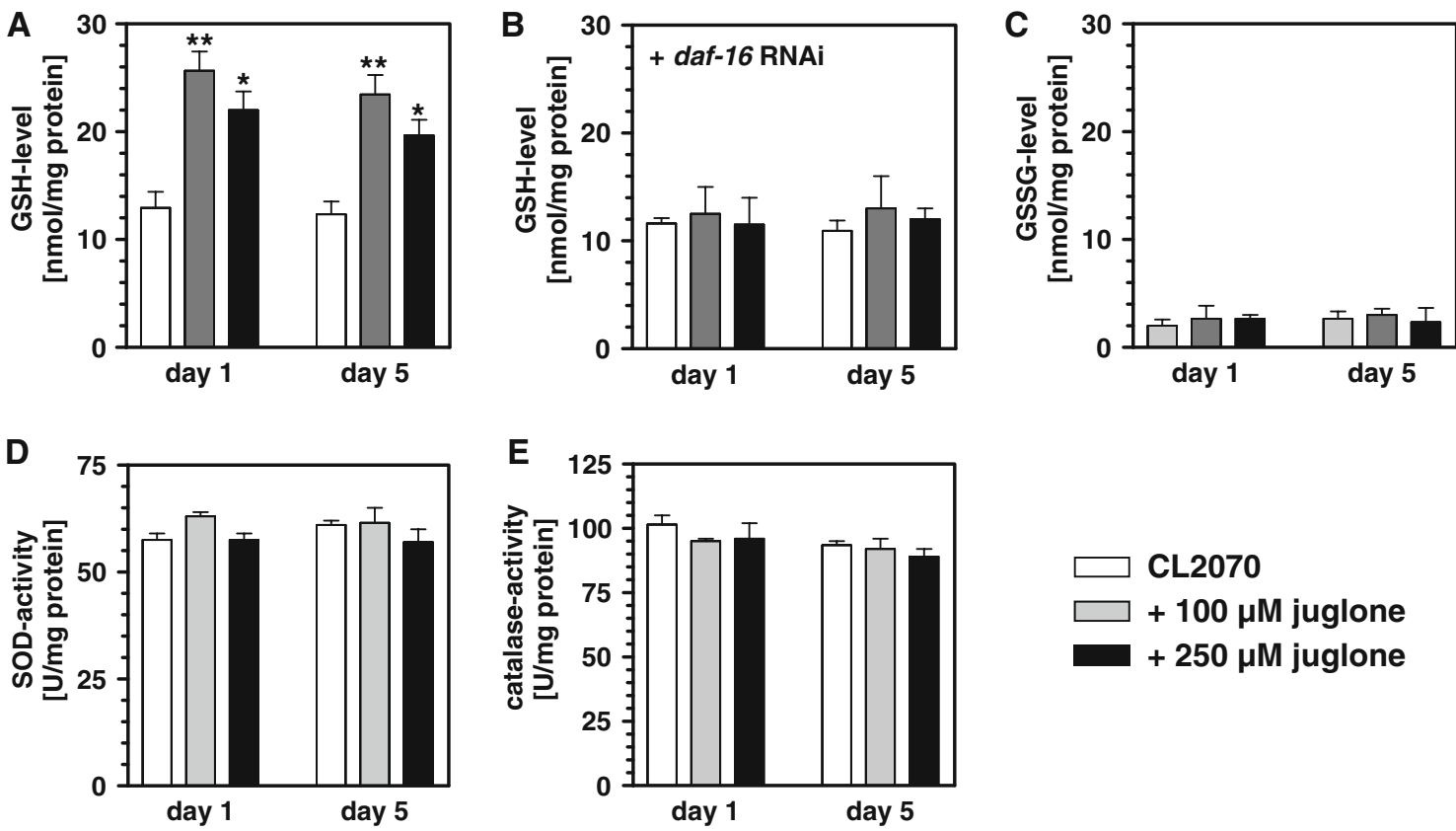

Fig. 4 GSH-levels but not SOD- or catalase activities are increased by juglone in CL2070. Levels of GSH were increased by the ROSgenerator (a) with $* P<0.05$, and $* * P<0.01$ versus the control, but DAF-16 RNAi inhibited the juglone-induced increase in GSH-levels

(b). GSSG-levels remained unaffected by juglone (c). SOD-activities (d) and catalase-activities (e) did not differ significantly between untreated CL2070 or those treated with 100 or $250 \mu \mathrm{M}$ juglone

A

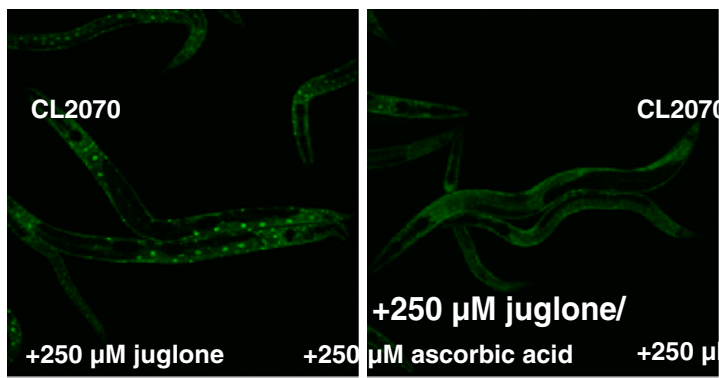

C

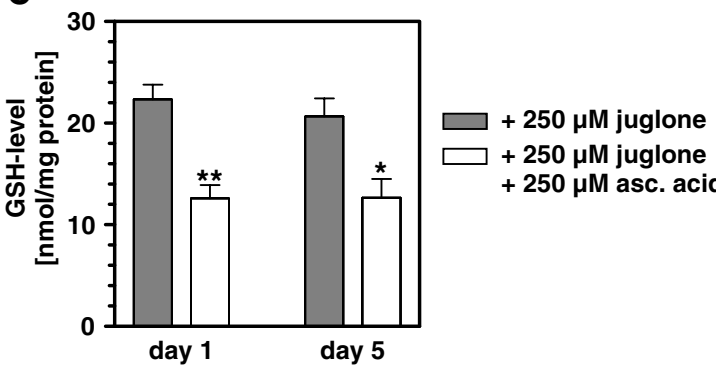

Fig. 5 Ascorbic acid prevents juglone-induced effects in CL2070. Ascorbic acid at $250 \mu \mathrm{M}$ blocks nuclear translocation of DAF-16 in TJ356 (a), and in CL2070 hsp-16.2p::GFP expression (b), increase in GSH-levels (c), and reductions in survival under heat-stress (d), that

in CL2070 and this response was blocked by DAF-16 RNAi. Since GSSG-levels remained unaltered by juglonetreatment our results suggest that the increased GSH-levels
B
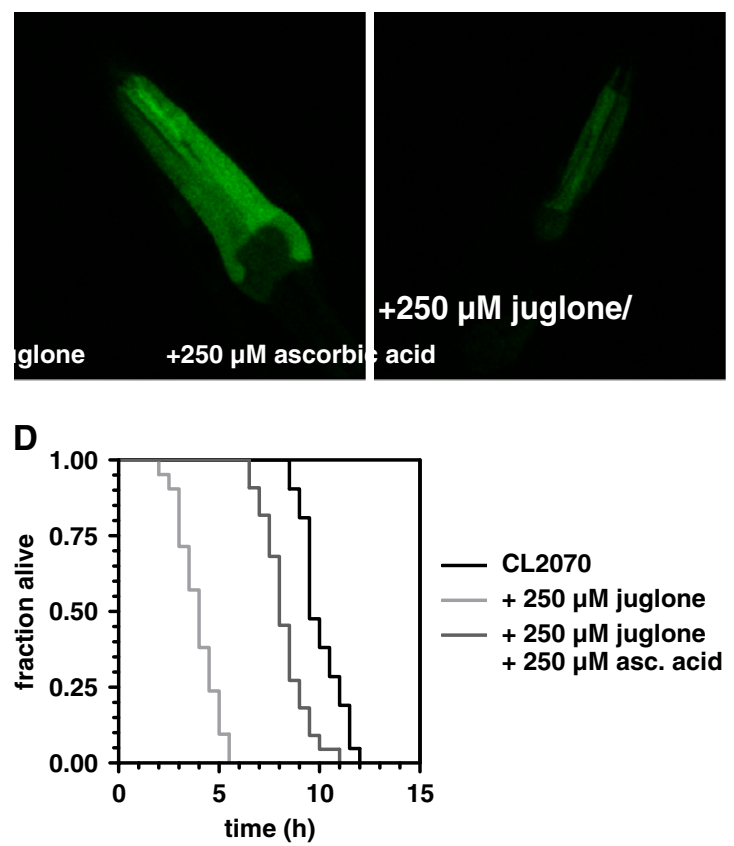

were all caused by $250 \mu \mathrm{M}$ juglone. $* * * P<0.001$ versus juglonetreated nematodes. $P$-value for significance of difference of survival curve of CL2070 treated with $250 \mu \mathrm{M}$ juglone plus $250 \mu \mathrm{M}$ ascorbic acid and those treated with $250 \mu \mathrm{M}$ juglone alone was $P<0.0001$

are due to de novo synthesis. Known other target genes of DAF-16 such as sod-3 or ctl-1 however did not show any changes in enzyme activities in response to juglone 
treatment. Recently it was shown that suppression of sod-3 expression in $C$. elegans has no or only modest effects on longevity [24, 25]. This is surprising since in long living age- 1 mutants, in which interrupted insulin signaling causes hyperresistance to oxidative stress both, SOD and catalase, showed an age-dependent increase that was not seen in the parental strain [19]. But other studies also failed to demonstrate changes in gene expression of both antioxidative enzymes in response to a short-term exposure to $90 \%$ oxygen in wildtype, daf-16 or age-1 mutants. This suggest as our data also indicate that SOD and catalase do not play a prominent role in the adaptive response to oxidative stress [36]. That, however, the responses measured in C. elegans are indeed dependent on oxidative stress was demonstrated by applying ascorbic acid in order to scavenge the ROS produced by juglone. Ascorbic acid prevented all of the juglone-induced adaptations, and also the diminished stress resistance observed when CL2070 were preincubated with high concentrations of juglone.

In conclusion, our studies show that oxidative stress caused in $C$. elegans by intake of a ROS-generator via the oral route induces nuclear DAF-16 translocation that is followed by enhanced expression of small HSP-16.2 and an increase in GSH-levels. This adaptive response enables animals to maintain a normal lifespan until the ROS stress exceeds a certain threshold of adaptive protective capacity leading to acute toxicity and premature death.

Acknowledgments We thank the Caenorhabditis Genetics Center, University of Minnesota for supplying the Bristol N2, CL2070 and TJ356 strains. We acknowledge Mr. A. Stamfort for statistical advice. This work was supported by the BASF, Ludwigshafen, Germany.

\section{References}

1. Aebi H (1986) Catalase in vitro. Meth Enzymol 105:121-126

2. Avery L, Shtonda BB (2003) Food transport in the C. elegans pharynx. J Exp Biol 206:2441-2457

3. Brenner S (1974) The genetics of Caenorhabditis elegans. Genetics 77:71-94

4. Blum J, Fridovich I (1983) Superoxide, hydrogen peroxide, and oxygen toxicity in two free-living nematode species. Arch Biochem Biophys 222:35-43

5. Clark J, Ding S (2006) Generation of RNAi libraries for highthroughput screens. J Biomed Biotechnol 2006:1-7

6. de Jong WW, Caspers GJ, Leunissen JA (1998) Genealogy of the alpha-crystallin — small heat-shock protein superfamily. Int J Biol Macromol 22:151-162

7. Garigan D, Hsu AL, Fraser AG, Kamath RS, Ahringer J, Kenyon C (2002) Genetic analysis of tissue aging in Caenorhabditis elegans: a role for heat-shock factor and bacterial proliferation. Genetics 161:1101-1112

8. Hekimi S, Guarente L (2003) Genetics and the specificity of the aging process. Science 299:1351-1354

9. Houthoofd K, Braeckman BP, Johnson TE, Vanfleteren JR (2003) Life extension via dietary restriction is independent of the
Ins/IGF-1 signalling pathway in Caenorhabditis elegans. Exp Gerontol 38:947-954

10. Houthoofd K, Braeckman BP, Lenaerts I, Brys K, De Vreese A, Van Eygen S, Vanfleteren JR (2002) Axenic growth up-regulates mass-specific metabolic rate, stress resistance, and extends life span in Caenorhabditis elegans. Exp Gerontol 37:1371-1378

11. Houthoofd K, Braeckman BP, Lenaerts I, Brys K, Matthijssens F, De Vreese A, Van Eygen S, Vanfleteren JR (2005) DAF-2 pathway mutations and food restriction in aging Caenorhabditis elegans differentially affect metabolism. Neurobiol Aging 26: 689-696

12. Hsu AL, Murphy CT, Kenyon C (2003) Regulation of aging and age-related disease by DAF-16 and heat-shock factor. Science 300:1142-1145

13. Ji LL (1999) Antioxidants and oxidative stress in exercise. Proc Soc Exp Biol Med 222:283-292

14. Jones D, Candido EP (1999) Feeding is inhibited by sublethal concentrations of toxicants and by heat stress in the nematode Caenorhabditis elegans: relationship to the cellular stress response. J Exp Zool 284:147-157

15. Jones D, Dixon DK, Graham RW, Candido EP (1989) Differential regulation of closely related members of the hsp16 gene family in Caenorhabditis elegans. DNA 8:481-490

16. Kamath RS, Martinez-Campos M, Zipperlen P, Fraser AG, Ahringer J (2001) Effectiveness of specific RNA-mediated interference through ingested double-stranded RNA in Caenorhabditis elegans. Genome Biol 2:RESEARCH0002

17. Kenyon C, Chang J, Gensch E, Rudner A, Tabtiang R (1993) A C. elegans mutant that lives twice as long as wild type. Nature 366:461-464

18. Lamitina ST, Strange K (2005) Transcriptional targets of DAF-16 insulin signaling pathway protect $C$. elegans from extreme hypertonic stress. Am J Physiol 288:C467-C474

19. Larsen PL (1993) Aging and resistance to oxidative damage in Caenorhabditis elegans. Proc Natl Acad Sci 90:8905-8909

20. Link CD, Cypser JR, Johnson CJ, Johnson TE (1999) Direct observation of stress response in Caenorhabditis elegans using a reporter transgene. Cell Stress Chaperones 4:235-242

21. Link CD, Taft A, Kapulkin V, Duke K, Kim S, Fei Q, Wood DE, Sahagan BG (2003) Gene expression analysis in a transgenic Caenorhabditis elegans Alzheimer's disease model. Neurobiol Aging 24:397-413

22. Marklund S, Marklund G (1974) Involvement of the superoxide anion radical in the autoxidation of pyrogallol and a convenient assay for superoxide dismutase. Eur J Biochem 47:469-474

23. Mehlen P, Kretz-Remy C, Preville X, Arrigo AP (1996) Human hsp27, Drosophila hsp27 and human alpha B-crystallin expression-mediated increase in glutathione is essential for the protective activity of these proteins against TNFalpha-induced cell death. EMBO J 15:2695-2706

24. Murphy CT (2006) The search for DAF-16/FOXO transcriptional targets: approaches and discoveries. Exp Gerontol 41:910-921

25. Murphy CT, McCarroll SA, Bargmann CI, Fraser A, Kamath RS, Ahringer J, Li H, Kenyon C (2003) Genes that act downstream of DAF-16 to influence the lifespan of Caenorhabditis elegans. Nature 424:277-283

26. Olsen A, Vantipalli MC, Lithgow GJ (2006) Lifespan extension of Caenorhabditis elegans following repeated mild hormetic heat treatments. Biogerontology 7:221-230

27. Preville X, Salvemini F, Giraud S, Chaufour S, Paul C, Stepien G, Ursini MV, Arrigo AP (1999) Mammalian small stress proteins protect against oxidative stress through their ability to increase glucose-6-phosphate dehydrogenase activity and by maintaining optimal cellular detoxifying machinery. Exp Cell Res 247: $61-78$ 
28. Rahman I, Kode A, Biswas SK (2006) Assay for quantitative determination of glutathione and glutathione disulfide levels using enzymatic recycling method. Nat Protoc 1:3159-3165

29. Rea SL, Wu D, Cypser JR, Vaupel JW, Johnson TE (2005) A stress-sensitive reporter predicts longevity in isogenic populations of Caenorhabditis elegans. Nat Genet 37:894-898

30. Schaffitzel E, Hertweck M (2006) Recent aging research in Caenorhabditis elegans. Exp Gerontol 41:557-563

31. Söti C, Csermely P (2007) Protein stress and stress proteins: implications in aging and disease. J Biosci 32:511-515

32. Stiernagel T (1999) Maintenance of C. elegans. In: Hope IA (ed) C. elegans: a practical approach, vol 281. Oxford University press, Oxford, pp 21

33. Szewczyk NJ, Udranszky IA, Kozak E, Sunga J, Kim SK, Jacobson LA, Conley CA (2006) Delayed development and lifespan extension as features of metabolic lifestyle alteration in C. elegans under dietary restriction. J Exp Biol 209:4129-4139

34. Valdez MM, Clark JI, Wu GJ, Muchowski PJ (2002) Functional similarities between the small heat shock proteins Mycobacterium tuberculosis HSP 16.3 and human alphaB-crystallin. Eur J Biochem 269:1806-1813

35. Walker GA, Lithgow GJ (2003) Lifespan extension in C. elegans by a molecular chaperone dependent upon insulin-like signals. Aging Cell 2:131-139

36. Yanase S, Yasuda K, Ishii N (2002) Adaptive responses to oxidative damage in three mutants of Caenorhabditis elegans (age-1, mev-1 and daf-16) that affect life span. Mech Ageing Dev 123: $1579-1587$ 